\title{
Gastrointestinal Mucormycosis: A Challenge during COVID-19 Pandemic
}

\author{
Jagdish Chander
}

\begin{abstract}
In India, the second wave of COVID-19 pandemic came with unprecedented number of patients in the months of April, May, and June, 2021. This time correspondingly there was a record number $(50,000)$ of mucormycosis, which was popularized by the mainstream media as "black fungus." The most common presentation was rhino-orbital mucormycosis followed by pulmonary and gastrointestinal mucormycosis. Scientifically this disease should be termed as COVID-19-associated mucormycosis (CAM). The Government of India had to declare it as an emergency situation under the Epidemic Diseases Act, 1897, on May 20, 2021. A large number of professional bodies in medical sciences, particularly in the field of microbiology, came out with various guidelines to tackle the challenging issue. The most common species involved is Rhizopus arrhizus, which is found in our surroundings, food material, and different places. Sometimes it is taken as a contaminant or "bread-mold" in houses or "lid-lifter" in the laboratory. But when there is an underlying background of any disease like diabetic mellitus, the same very fungus also becomes life-threatening. During this intervening period of second and looming third wave, patients are presenting with recurrence of the disease. The diagnosis is not very difficult when the index of suspicion is high. The direct finding of nonseptate hyphae with right-angle branching followed by cultural confirmation on Sabouraud Dextrose Agar clinch the final diagnosis. As far as the treatment is concerned, it is mainly surgical intervention of the necrotic tissue followed by antifungals like amphotericin B (conventional/liposomal), posaconazole, isavuconazole, apart from taking care simultaneously of the underlying risk factors like diabetes mellitus. With timely management the patient can be saved easily otherwise it can prove fatal.
\end{abstract}

Keywords: Amphotericin B, CAM, COVID-19, Gastrointestinal mucormycosis, Pandemic.

Journal of Gastrointestinal Infections (2021): 10.5005/jp-journals-10068-3052

\section{INTRODUCTION}

During the last four decades, fungal infections are increasingly being reported among the population at large. Some of them, i.e., candidiasis, cryptococcosis, pneumocystosis, talaromycosis, aspergillosis, etc., were frequently encountered after the onset of AIDS pandemic in 1980s to the extent that they were designated as AIDS-defining illnesses. However, lately, another infection was also added to this opportunistic fungal list, not going parallel to earlier ones, that is, mucormycosis. Although rare but one of its clinical components is gastrointestinal (GI) manifestation. Majority of the presenting cases are rhino-orbital, a few belong to pulmonary type followed by GI mucormycosis. The main disease itself, along with GI tract manifestations, has posed a very serious challenge for the medical fraternity during this on-going COVID-19 pandemic.

The media has branded the disease as "black fungus," which is essentially a misnomer as there is designated category of "black fungi" already existing in the medical literature causing tinea nigra, chromoblastomycosis, pheohyphomycosis, etc. The deciding factor for any "black fungus" is the presence of melanin in the fungal cell wall otherwise they are considered as colorless, i.e., hyaline and the Mucorales are definitely hyaline fungi. There is no end to such unfounded and fancy nomenclatures. Today it is a "white fungus" or "yellow fungus" and tomorrow there will be red, blue, or green fungi, which should be discouraged. Preferably, it should be called as COVID-19-associated mucormycosis.

The understanding about Mucormycetes has evolved as these are now emerging as highly pathogenic organisms invariably entailing fatal consequences, especially when an obvious underlying predisposing factor like diabetes mellitus already exists
Fungal Clinic, Panchkula, Haryana, India,

Corresponding Author: Jagdish Chander, Fungal Clinic, Panchkula, Haryana, India, e-mail: jchander@hotmail.com

How to cite this article: Chander J. Gastrointestinal Mucormycosis: A Challenge during COVID-19 Pandemic. J Gastrointest Infect 2021; 11:30-35.

Source of support: Nil

Conflict of interest: None

in a particular clinical setting. The cases are increasingly reported among absolutely healthy individuals also where there is no obvious underlying risk factor. Unlike other fungal diseases, even the AIDS pandemic could not significantly affect the incidence as well as prevalence of mucormycosis during the last four decades; however, an upsurge in the number of diabetic patients has really changed the entire scenario more or less like an epidemic in the Southeast Asian Subcontinent by posing a very serious health threat. The present COVID-19 or the future pandemics will aggravate the situation in the times to come.

The so-called "black fungus" disease, i.e., mucormycosis is in general an acute infection caused by several agents belonging to phylum Glomeromycota. These saprotrophic fungi are found ubiquitously in the atmosphere, i.e., soil and environment, in our surroundings. Earlier these mucoralean fungi used to be taken as nonpathogenic to man and animals. Rather they were considered as merely fungal contaminants in the diagnostic microbiology laboratory. During the first wave of COVID-19, these presentations were not observed; however, in the second

() The Author(s). 2021 Open Access This article is distributed under the terms of the Creative Commons Attribution 4.0 International License (https://creativecommons. org/licenses/by-nc/4.0/), which permits unrestricted use, distribution, and non-commercial reproduction in any medium, provided you give appropriate credit to the original author(s) and the source, provide a link to the Creative Commons license, and indicate if changes were made. The Creative Commons Public Domain Dedication waiver (http://creativecommons.org/publicdomain/zero/1.0/) applies to the data made available in this article, unless otherwise stated 
wave about 50,000 cases were reported, half of them being lifethreatening. Now, the third wave is also looming, which may lead to alarming consequences. The upsurge in cases is because of the COVID-19 pandemic, contributed by various risk factors. In the present review, description of the causative agent, upsurge of mucormycosis during COVID-19 pandemic, virulence, clinical manifestations, diagnosis, and treatment of the disease have been discussed in detail.

\section{What is Mucormycosis?}

The nomenclature, mucormycosis, is an umbrella term used for diseases caused by many nonseptate filamentous fungi. Classified under orders Mucorales and Entomophthorales, they were previously considered members of phylum Zygomycota, which are elevated to the rank of subphylum Mucoromycotina and Entomophthoromycotina, respectively, under phylum Glomeromycota. The phylum Zygomycota is now abandoned, hence, consequently the term zygomycosis also no longer exists. Some investigators prefer to call the disease caused by members belonging to subphylum Mucoromycotina as mucormycosis and to subphylum Entomophthoromycotina as entomophthoromycosis or even subcutaneous mucormycosis, which is further subdivided into two clinical types, i.e., conidiobolomycosis and basidiobolomycosis. ${ }^{1}$

The Mucormycete is a group of lower fungi and their hyphae are generally nonseptate. However, when these septa occur they are solid cross-walls with no pores and there is no flow of cytoplasmic material between adjoining cells. These reproduce asexually by way of sporangiospores formed within a sac called as sporangium and/ or by means of conidial development. These fungi also reproduce sexually by formation of a single, dark, thick-walled spore called zygospore.

These fungi are seen as broad, nonseptate hyphae in tissue with a strong predilection to invade blood vessels due to their angioinvasive nature thereby leading to extensive necrosis of the surrounding area eventually formation of embolism. Since the morphology of these organisms is indistinguishable in histopathological sections, culture is essential for the exact identification of the causative Mucorales species. The course and outcome of disease differ according to anatomical site involved as well as the nature of fungal species isolated in a particular patient.

The fungi are found in food items, soil, air, and may be frequently encountered as laboratory contaminant. The spores are widely distributed, growing on leaf litter and other decaying carbohydrate substrates. The spores are found in large number in damp interiors and around composting vegetation and even present as "bread molds" appearing as grayish, fluffy, and rapidly spreading growth. Because of their rapid growth and prolific spore-forming capacity, inhalation of conidia is a routine occurrence. It is now the third most common invasive mycoses after candidiasis and aspergillosis.

\section{Upsurge of Mucormycosis Cases}

The fundamental question is why there is an upsurge of cases of mucormycosis during this pandemic of COVID-19 including the GI tract manifestations. This situation was not so grim during its first wave in 2020 but all of a sudden, like a sort of tsunami, within a short span of time, many cases were reported all over the country. This began with a few case reports from Ganga Ram Hospital, New Delhi, and almost simultaneously from Gujarat. Subsequently, cases started pouring in from almost every nook and corner of the country. Most of the patients had already recovered from COVID-19 episode and therefore discharged; however, some of them came back to the hospital with a fulminant disease. The second wave of COVID-19 has now declined but recurrence is common among the already treated cases. The various risk factors are contemplated and evidence-based studies need to be carried out before reaching a definitive conclusion.

First and foremost, there is injudicious use of steroids during the course of the management of COVID-19 and that too in high doses than the prescribed ones for a prolonged duration. The other risk factors may be like prolonged use of contaminated masks, without periodically changing as per the prescribed guidelines. There is a strong possibility of use of contaminated accessory while inhaling oxygen among patients. During the second wave of COVID-19 pandemic, there was an acute shortage of medical oxygen in the country hence most of the hospitals had to shift on to the industrial oxygen. Although it is said that there is no fundamental difference between both the grades of oxygen except for a minor one of dispensing but the public at large still suspect it to be one of the causes. The humidifiers used while delivering the oxygen, i.e., distilled water vs sterile or tap water is also suspected to be the source. Moreover, frequent and excessive steam inhalation is also considered as one of the risk factors.

Among other suspected risk factors, there is a higher level of iron in the form of ferritin among the COVID-19 patients, which is also favorable for copious growth of Mucormycetes. In addition almost all patients of COVID-19 are taking zinc as a preventive and/ or therapeutic agent, which is also conducive to the fungal growth. This fungal infection also affects people who are already on some medication like prolonged use of antibiotics. Some of the reports state that the delta variant of the SARS-CoV-2 (B.1.617.2) is targeting the islets of Langerhans in the pancreas through ACE2 receptors, jeopardizing insulin production thereby leading to diabetic state among patients during their recovery phase.

Mucormycosisis declared as a notifiable disease by various Indian states beginning with Rajasthan. Eventually Indian Council of Medical Research (ICMR)/Government of India (GOI) also announced this disease under Epidemic Diseases Act, 1897, on May 20, 2021, wherein all government and private health facilities and medical colleges are to follow guidelines for its screening, diagnosis, and management. The advisory goes on to say that mucormycosis, if remain uncared, may turn out to be fatal. Hence to prevent the disease, blood glucose level should be regularly monitored during the post-Covid period. Consequently, all cases reported are to be conveyed to the World Bank-funded Integrated Disease Surveillance Project of the respective States or Union Territories.

In addition to the NCDC/ICMR/GOI, Fungal Infection Study Forum, European Confederation of Medical Mycology (ECMM), and International Society for Human and Animal Mycology (ISHAM) have also released their respective guidelines on this acutely emerging disease during the COVID-19 pandemic.

\section{Virulence of Mucormycosis}

The virulence of these fungi is simply of low order and usually sporadic infections occur throughout the world particularly in severely debilitated patients. Being an opportunistic infection, mucormycosis is produced by contaminant fungi in a host whose immunological defense mechanisms are weakened by endogenous causes like uncontrolled diabetes mellitus in Southeast Asia whereas malignancy, leukemia, or exogenous causes like immunosuppressive therapy in the Western world. 
The breakthrough mucormycosis cases are also reported among patients with hematologic malignancies receiving hematopoietic stem cell transplants or intensive chemotherapy, given voriconazole for preventing aspergillosis or have undergone COVID-19 vaccination.

The neutrophils appear to play a major role in the defense of host from infection caused by Mucormycetes. In normal hosts, macrophages prevent initiation of infection by phagocytosis and oxidative killing of spores. On the other hand, among hosts with uncontrolled diabetes mellitus and other underlying factor, monocytes/macrophages are dysfunctional and fail to suppress spore germination process. The hosts with neutrophil defects, either qualitative or quantitative, are predisposed to infection by fungal agents of this class.

Similar to aspergillosis, phagocytes, polymorphonuclear neutrophils, and macrophages play a significant role among patients of mucormycosis. Infection can occur by inhalation, percutaneous inoculation, or ingestion. The spores are inhaled into lungs where they are ingested by alveolar macrophages. These cells are known to inhibit the germination of ingested spores to some extent but their activity to kill them is limited. Further, when they evade antifungal activity of macrophages and germinate into mycelial form, polymorphonuclear neutrophils and peripheral monocytes are expected to work against fungi. The former, which possess property of fungicidal activities against Mucormycetes, are known to play an important role in defense against mucormycosis; therefore, leukocytopenic patients are extremely susceptible. The increased risk of mucormycosis in patients with ketoacidosis may also be due to the release of iron bound to proteins. Due to ketoacidosis, low serum $\mathrm{pH}$ diminishes the phagocytic effect of macrophages, chemotactic, and oxidative burst of neutrophils.

The most common encountered mucormycete, Rhizopus arrhizus, has several virulence factors like angioinvasive nature, growth at or above body temperature, production of destructive enzymes, dormant spores which are resistant to destruction at extremes of temperature along with active ketone reductase system and hydroxamate siderophores. The diabetic patients with ketoacidosis are usually more affected by mucormycosis. Rhizopus species have an active ketone reductase system hence thrive in high glucose and acidotic conditions. These patients also have decreased phagocytic activity because of an impaired glutathione pathway. The exact mechanism of increased susceptibility among these patients remains somewhat unknown, probably a combination of metabolic abnormalities present in the patients with diabetes. Hyperglycemia or acidosis alone does not permit fungal growth in vivo although acidosis without hyperglycemia is reported with invasive mucormycosis. The normal serum inhibits Rhizopus species whereas serum from patients of diabetic ketoacidosis stimulates its growth.

This has been observed that patients, on dialysis and iron overload, who are being treated with deferoxamine, an iron chelator, are more susceptible to mucormycosis. It is probably because Mucorales use this chelator as a siderophore to obtain more iron. The other risk factors include neutropenia, high-dose systemic steroids, protein-calorie malnutrition, solid organ and bone marrow transplants, immunodeficiency, leukemia and intravenous drug abusers who may inject spores of Mucorales with the drugs and then present with space-occupying lesions of the central nervous system.

\section{Clinical Manifestations of Mucormycosis}

As such the nomenclature as mucormycosis comes from Mucorales and not from its one of its genera, i.e., Mucor, which is confused. Irrespective of the genus or species, based on the sites involved, the disease is called mucormycosis, which presents as the following six clinical categories. ${ }^{2}$

The clinical manifestations are (i) rhino-orbito-cerebral, (ii) pulmonary, (iii) cutaneous, (iv) gastrointestinal, (v) isolated renal, and (vi) disseminated mucormycosis.

Each category is associated with certain underlying disorders, relationship, and existence of variable factors. The commonest type is rhino-orbito-cerebral with almost $50 \%$ mortality and $R$. arrhizus is the usual causative agent. ${ }^{3,4}$

The signs and symptoms of GI mucormycosis are nonspecific and vary considerably depending on the exact site as well as the extent of involvement. These include abdominal pain, distension, vomiting, diarrhea, hematemesis, and melena. The patients on dialysis are reported to develop the disease as most of them are treated with desferrioxamine (deferoxamine and desferal) used as chelator for either iron or aluminum and may be for both. ${ }^{5}$

The necrotic and gangrenous lesions of intestine are rarely reported among the COVID-19 patients due to GI manifestations of mucormycosis. This may be a primary disease where there is no involvement of other systems. However, mostly it is found to be presenting with rhino-orbital or disseminated infections. Simultaneous involvement of renal and GI tract is also reported. As such Gl type occurs in general accounting for about $7 \%$ of all cases of mucormycosis, most often involving the stomach. It is primarily found among patients suffering from extreme malnutrition and is acquired by ingesting food contaminated with fungal spores. Moreover, ingestion of fermented milk, porridge, and alcohol made from corn and herbal products have been implicated in GI mucormycosis. The lesions in stomach are followed by colon, ileum, and esophagus. This may follow surgery from filthy trauma to abdomen or contaminated ileostomy. Ulceration of gastric mucosa with thrombosis of associated vessels has been observed (Figs 1 and 2). The disease is usually found in adult patients but has also been reported in neonates, low-birth weight infants, and young children. ${ }^{6}$

The digestive system in mucormycosis is primarily involved in patients suffering from malnutrition. The manifestations range

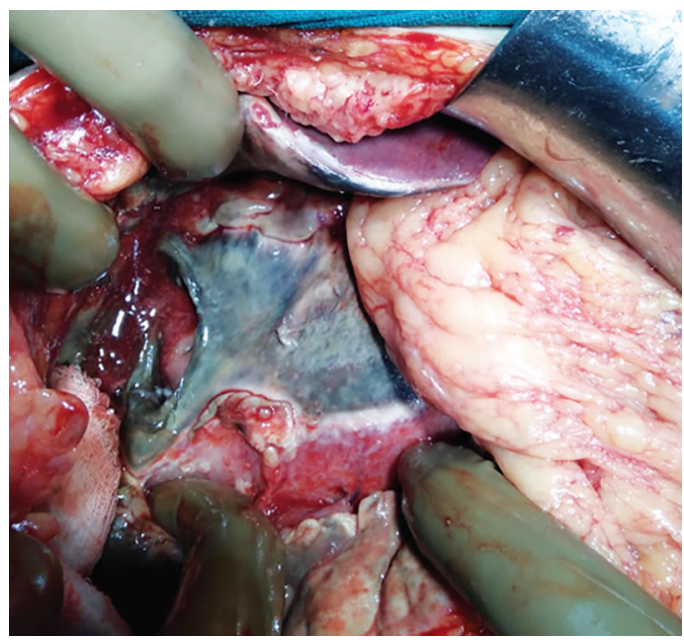

Fig. 1: Extensive necrotizing gastric lesions due to mucormycosis over the pyloric end of the stomach of a patient 


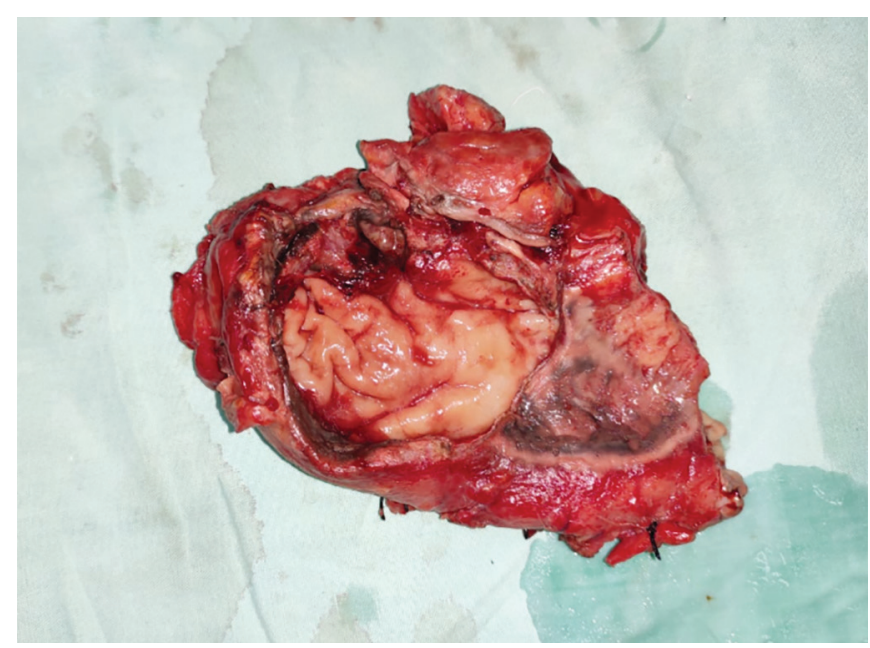

Fig. 2: Extensive necrotizing gastric lesions surgically removed from the pyloric end of the stomach of a patient

from mere colonization of peptic ulcers to infiltrative disease with vascular invasion and dissemination. It can be categorized into three forms: colonization, infiltration, and vascular invasion. Colonization usually occurs in preexisting gastric ulcers and is not fatal. Invasive form of gastric mucormycosis has variable presentation and is usually fatal. This form can either invade preexisting peptic ulcer or invade the stomach de novo. The disease usually presents as epigastric discomfort, Gl bleeding, or viscous perforation in patients with established predisposing factors. In the invasive variant, there is involvement of vessel walls producing thrombosis, hemorrhage, necrosis, and ulceration of local tissue, and usually has a fatal outcome. Intestinal tract involvement is relatively rare with terminal ileum, cecum, and colon being primarily affected sites. ${ }^{7}$

\section{Diagnosis of GI Mucormycosis}

Earlier, diagnosis of gastric mucormycosis used to be made on the basis of postmortem examination and was rarely possible during life. More recently, radiological imaging and endoscopic biopsies have established diagnosis at an earlier stage during life, allowing attempt to a successful treatment. Gastric brushing is less invasive than biopsy and allows early identification of organisms as compared to histopathological processing. A very similar type of disease, GI basidiobolomycosis, is caused by Basidiobolus ranarum, members of order Entomophthorales, which is a rare clinical entity and about 80 cases have been reported in the literature. It is usually found in Middle East countries like Iran, Saudi Arabia, Kuwait, Oman, etc., but occasionally reported from India also. ${ }^{8}$

The patient with $\mathrm{Gl}$ mucormycosis is often misdiagnosed as having an intra-abdominal abscess. It is usually confused with GI basidiobolomycosis, other inflammatory bowel diseases, malignancies, appendicitis, and diverticulitis. GI basidiobolomycosis involves the stomach, small intestine, and colon; however, it can disseminate to the liver, pancreas, and renal system. This may also present with complications like bowel perforation, obstructive uropathy, esophageal varices, duodenobiliary fistula, or even death. The histopathological examination is useful in establishing the accurate diagnosis, which reveals Splendore-Hoeppli phenomenon; however, culture remains the gold standard to diagnose GI basidiobolomycosis. $^{9}$
The clinician should keep high index of suspicion about mucormycosis, which is necessary in post-COVID-19 patients presenting with mesenteric ischemia or bowel perforation especially if they were diabetic or have used high-dose steroids. It is established that computed tomography scan and particularly magnetic resonance imaging are most helpful in enabling an early detection of orbital, sinus, meningeal, intraparenchymal, cerebral lesions, intracranial vascular occlusion as well as $\mathrm{Gl}$ involvement even before full-fledged clinical signs develop. These imaging techniques are helpful in defining the extent of soft tissue involved and are more useful in planning surgical intervention in addition to establishing the diagnosis. The COVID-19 case may present with markedly increased inflammatory cytokines like IL-2, IL-6, IL-10, and tumor necrosis factor-alpha, impaired cell-mediated immunity, both CD4+ and CD8+ T cells.

In the laboratory, a thorough understanding of GI mucormycosis is of paramount importance to establish an early diagnosis thereby appropriate treatment. The diagnosis is challenging and that too in COVID-19 times because of rapid fulminating course of disease and doubtful significance of isolates, which are commonly encountered as laboratory contaminants. One has to compete with the race of time. Therefore, detection of fungus in tissues is supplemented to establish significance of cultural isolate. The necrotic clinical materials from infected site may contain fungal elements; however, deeper tissue section may also be required. Detection of galactomannan or $\beta$-glucans in patients' serum, which is often helpful in diagnosis of other systemic fungal infection like aspergillosis, is of little value because Mucormycetes do not produce substantial amount of both these biomarkers.

The microscopic examination of biopsy material in potassium hydroxide/calcofluor-white (KOH/CFW) wet mount shows characteristic broad, nonseptate ribbon-like hyphae with rightangle branching at irregular intervals (Figs 3 and 4). These hyphae usually are very sparsely distributed in infected tissue, and therefore, histopathological stains are mandatory like hematoxylin and eosin (H\&E) (Fig. 5), Periodic acid-Schiff (PAS) (Fig. 6), and Gomori's methenamine silver. The inflammatory response is of neutrophilic nature and is associated with infarct, vascular, and perineural invasion. Sometimes, distorted hyphae are also seen in the tissues. The disease may also be established on the basis of fine-needle aspiration cytology from the affected site. Frozen

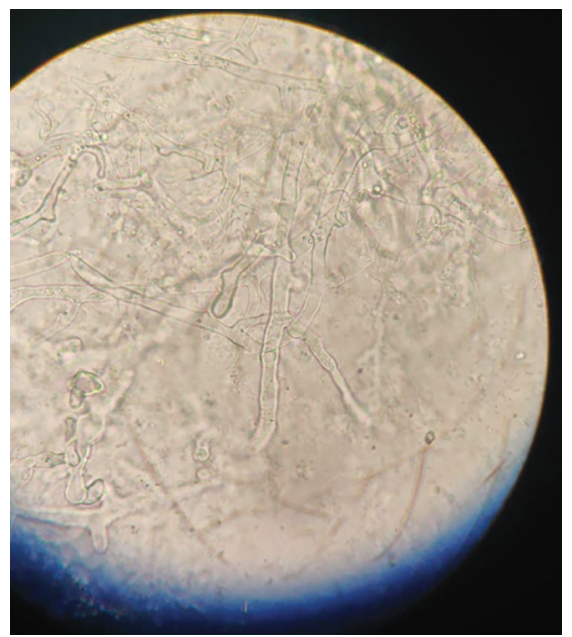

Fig. 3: Broad, ribbon-like, nonseptate hyphae with right-angle branching seen in wet mount $(\mathrm{KOH} \times 400)$ 


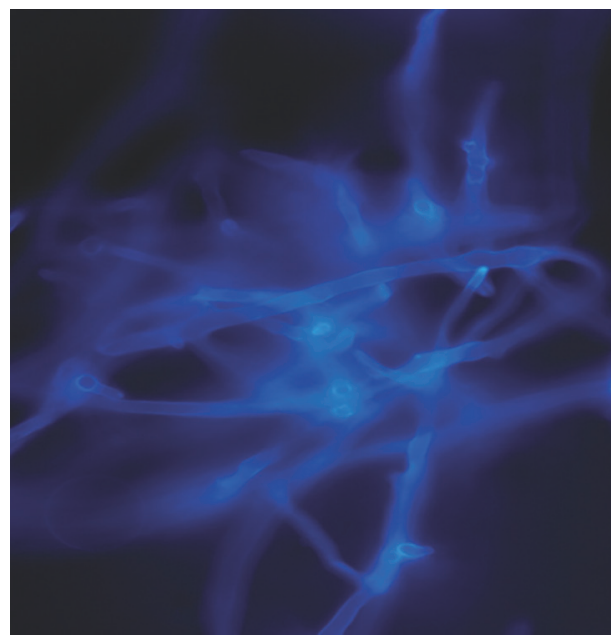

Fig. 4: Broad, ribbon-like, nonseptate hyphae with right-angle branching seen in wet mount $(C F W \times 400)$

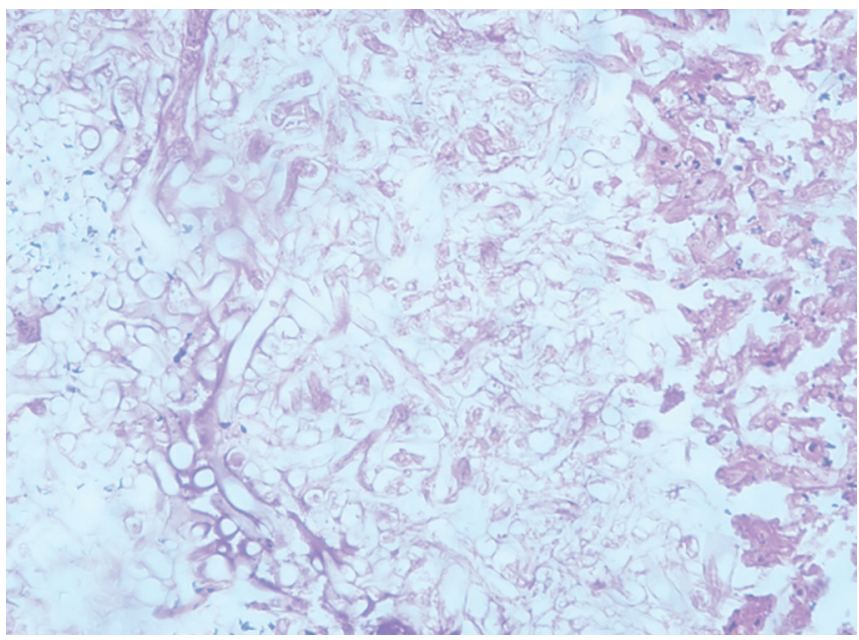

Fig. 5: Nonseptate hyphae of Mucormycetes with right-angle branching in a tissue sections $(\mathrm{H} \& \mathrm{E} \times 400)$

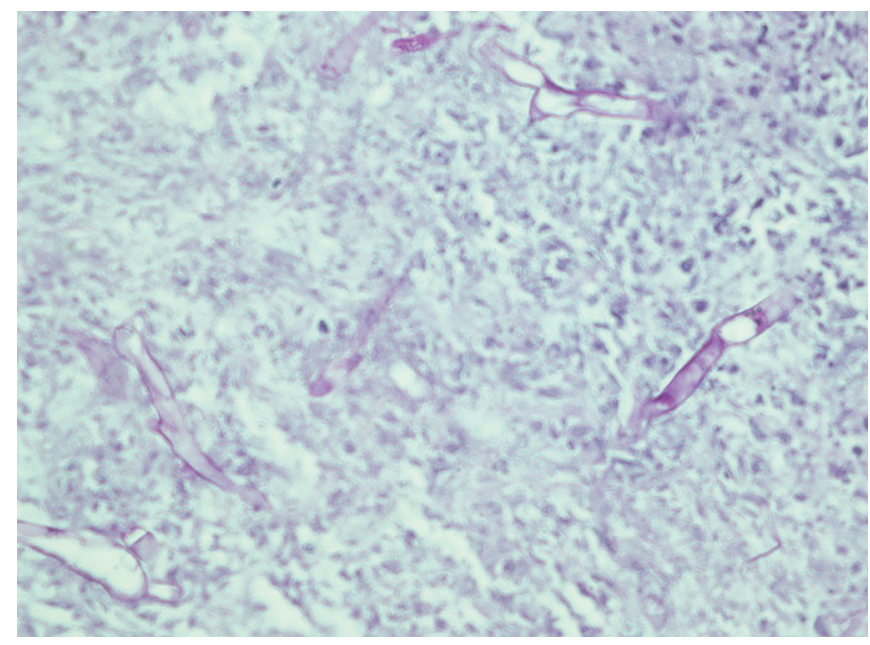

Fig. 6: Nonseptate hyphae of Mucormycetes with right-angle branching in a tissue sections (PAS $\times 400)$ section may be useful when operative procedure was contemplated without suspicion of $\mathrm{GI}$ mucormycosis.

The hyphal elements of Mucormycetes are recognized as nonseptate thick-walled (10-20 $\mu \mathrm{m})$ hyphae, with right-angle branching. Due to the absence of cross-walls, fluids from hyphae are free to escape during handling of tissues, hyphae collapse, and crinkle giving characteristic ribbon-like appearance. They do not radiate from a single point in tissue. These features distinguish them from slender hyphae of Aspergillus species, which have regular dichotomous branching at acute angle with frequent septation. Therefore, it is recommended not to homogenize tissue material and specimens must be as such directly inoculated on to culture media to keep texture intact thereby viability of fungal cells.

The Mucormycetes can be easily grown on conventional media like Sabouraud dextrose agar with antibiotics at both temperatures, i.e., 25 and $37^{\circ} \mathrm{C}$ but without cycloheximide, as it is inhibitory to most of them. Although the Mucormycetes are not fastidious fungi, still sometimes they fail to grow during primary isolation due to careless handling of specimen. Therefore, to avoid growth failure, some portion of the tissue may be kept in water added with a few drops of Yeast Malt Broth. In about $50 \%$ of cases there is no growth despite direct demonstration of the fungi. In such circumstance, molecular technique directly from the sample should be done to establish the diagnosis.

As mentioned the hyphal elements of Mucormycetes are prone to physical damage; therefore, specimens must be directly inoculated onto fungal culture media avoiding undue grinding. The relevance of isolates in clinical material may be difficult to establish if coenocytic hyphal elements are not seen in direct examination of $\mathrm{KOH} / \mathrm{CFW}$ wet mount or histopathological section. Hence repeated attempt to isolate organism from consecutive specimens provides strong evidence that isolate is clinically significant.

The rapidly growing mycelial colonies are floccose, dense, and hairy in appearance (Fig. 7). The mycelia are described as fibrous or with cotton-candy growth, which is very vigorous hence some of Mucormycetes of order Mucorales are called as "lid-lifters" as they press upon lid of petri dish from below. The isolate is identified on the basis of morphological features observed in lactophenol cotton blue (LCB) mount (Fig. 8) followed by molecular sequencing for confirmation. The antifungal susceptibility testing is also done to

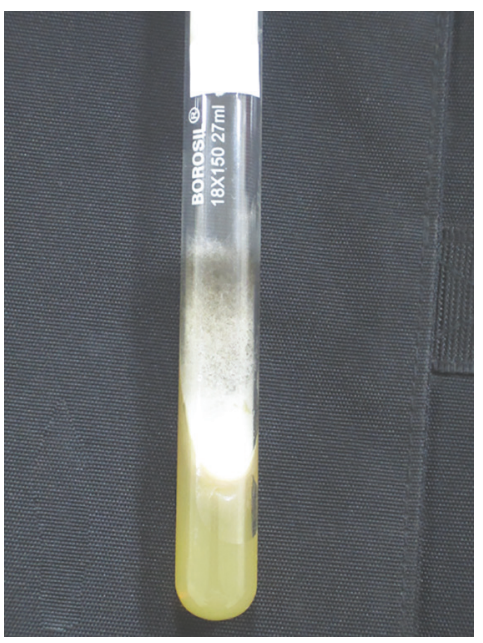

Fig. 7: Grayish mycelial growth of Rhizopus arrhizus in fungal culture tube after 3 days of incubation on Sabouraud dextrose agar at room temperature 


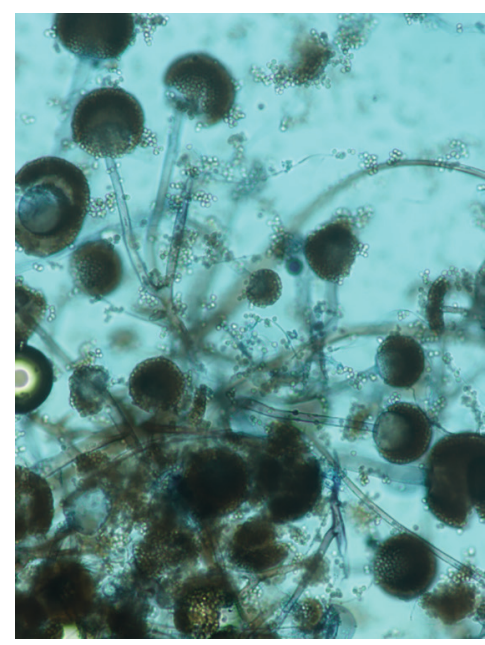

Fig. 8: Nonseptate hyphae of Rhizopus arrhizus with rhizoids, sporangia, and sporangiospores $(\mathrm{LCB} \times 400)$

establish whether the isolate is sensitive to a particular antifungal drug or not using CLSI or EUCAST methods. There is no reliable serological test for mucormycosis thereby cannot be recommended for the routine use in a diagnostic microbiology laboratory.

\section{Treatment}

During this pandemic of COVID-19, most of the national and international organizations like WHO, ISHAM, ECMM, FISF, and $\mathrm{NCDC/ICMR/GOI} \mathrm{have} \mathrm{issued} \mathrm{clinical} \mathrm{guidelines} \mathrm{for} \mathrm{the} \mathrm{diagnosis}$ and treatment of mucormycosis including the GI manifestations. The consensus is resultant from the experience of clinicians treating such cases. As GI mucormycosis is a life-threatening condition hence invariably it proves to be fatal within a very short span, particularly when specific diagnosis is not established well in time thereby leading to substantial delay in surgery as well as institution of proper antifungal regimen.

For treating the disease, first of all proper management of COVID-19 infection has to be instituted depending on its stage and severity, ensuring all the prescribed precautions. Try to avoid steroids therapy as far as possible; however, if at all indicated then regulated dose to be given for a minimum time period. Immediately remove all the risk factors like diabetes mellitus by starting insulin or other appropriate antidiabetic regimens. ${ }^{10}$

The therapeutic modalities can be divided into four concurrent approaches. These are (a) rapid correction of underlying predisposing condition of the host like diabetic ketoacidosis using insulin; (b) surgical debridement of necrotizing tissue for better circulation and penetration of drugs; (c) antifungal therapy; and (d) consideration of adjunctive treatment such as hyperbaric oxygen, if available. A combination of surgical debridement and antifungal drugs is required for an ideal treatment of mucormycosis. The temptation for small and frequent piecemeal debridement should be avoided, which further aggravates morbidity and mortality among the patients. It should be preferably done taking adequate surrounding healthy tissue for debridement. ${ }^{11}$

There are only three drugs available for treating $\mathrm{Gl}$ mucormycosis, i.e., intravenous amphotericin B (conventional/liposomal), posaconazole, and isavuconazole. It is observed to start with higher doses of antifungal drugs instead of stepwise increment. Drug resistance may be another hurdle but in case of majority of mucormycetes strains, these are found to be sensitive. Topical amphotericin B gel is a useful adjunct, which is applied locally after surgery.

The azole derivatives are not useful in treating GI mucormycosis due to lack of both in vivo and in vitro antifungal activity. However, exceptionally posaconazole and isavuconazole are found to be useful, which are available as oral and intravenous preparations. Some of the azole like voriconazole is otherwise counterproductive which enhances the disease process among mucormycosis patients. Similarly, echinocandins have no role in the treatment and there is no oral chemoprophylactic agent available for this fungal disease. Cytokines such as gamma interferon and granulocyte-macrophage colony-stimulating factors have also been used to treat GI mucormycosis.

The advisory guidelines, most of the times, prove to be powerless because there is nothing mandatory. Hence keeping in view the rapid and devastating course of mucormycosis, every institution should resort to its local mandatory protocol, wherein $\mathrm{KOH} / \mathrm{CFW}$ wet mount should be performed within half an hour of the arrival of suspected patient followed by surgical debridement within 2 hours in cases found to be positive. If this promptness is adopted, the patients may not require antifungal drugs also, which were in inordinate scarcity due to increased demand. However, comprehensive antifungal regimen is to be instituted as per the clinical and financial condition of the patient. If proper measures are not followed in letter and spirit one is bound to lose the patient of a treatable disease. All the COVID-19 patients should be made cautious at the time of discharge that they should check and regulate their sugar level at least for a period of few weeks.

\section{References}

1. Chander J, Kaur M, Singla N, et al. Mucormycosis: battle with the deadly enemy over a five-year period in India. J Fungi (Basel) 2018;4(2):46. DOI: 10.3390/jof4020046.

2. Chander J. Textbook of medical mycology. 4th ed. New Delhi: Jaypee Brothers Medical Publishers (P) Ltd; 2018.

3. Garg D, Muthu V, Sehgal IS, et al. Coronavirus Disease (COVID-19) associated mucormycosis (CAM): case report and systematic review of literature. Mycopathologia 2021;186(2):289-298. DOI: 10.1007/ s11046-021-00528-2.

4. Goel P, Jain V, Sengar M, et al. Gastrointestinal mucormycosis: a success story and appraisal of concepts. J Infect Public Health 2013;6(1):58-61. DOI: 10.1016/j.jiph.2012.08.004.

5. Jain $M$, Tyagi $R$, Tyagi $R$, et al. Post-COVID-19 gastrointestinal invasive mucormycosis. Indian J Surg 2021;1-3. DOI: 10.1007/s12262-021-03007-6.

6. Monte Junior ESD, Santos MELD, Ribeiro IB, et al. Rare and fatal gastrointestinal mucormycosis (zygomycosis) in a COVID-19 patient: a case report. Clin Endosc 2020;53(6):746-749. DOI: 10.5946/ce.2020.180.

7. Patra S, Vij M, Chirla DK, et al. Unsuspected invasive neonatal gastrointestinal mucormycosis: a clinicopathological study of six cases from a tertiary care hospital. J Indian Assoc Pediatr Surg 2012;17(4): 153-156. DOI: 10.4103/0971-9261.102329.

8. Pezzani MD, Di Cristo V, Parravicini C, et al. Gastrointestinal basidiobolomycosis: an emerging mycosis difficult to diagnose but curable. Case report and review of the literature. Travel Med Infect Dis 2019;31:101378. DOI: 10.1016/j.tmaid.2019.01.013.

9. Sharma A, Saxena R, Sinha A, et al. Disseminated gastrointestinal basidiobolomycosis (GIB) in an infant from Western India. Med Mycol Case Rep 2019;26:38-41. DOI: 10.1016/j.mmcr.2019.10.001.

10. Singh RP, Gupta N, Kaur T, et al. Rare case of gastrointestinal mucormycosis with colonic perforation in an immunocompetent patient with COVID-19. BMJ Case Rep 2021;14(7):e244096. DOI: 10.1136/ bcr-2021-244096.

11. Szarpak L, Chirico F, Pruc M, et al. Mucormycosis - a serious threat in the COVID-19 pandemic? J Infect 2021;83(2):237-279. S01634453(21)00257-7. DOI: 10.1016/j.jinf.2021.05.015. 